\title{
Inter-Patient Analysis of Tomographic Data
}

\author{
Christoph Busch \\ Computer Graphics Center \\ Wilhelminenstraße 7 \\ D-64283 Darmstadt \\ Tel: +496151 155236 \\ Fax: +496151155480 \\ E-mail: busch@igd.fhg.de
}

\begin{abstract}
This paper considers the computer-based support for the localization of pathological tissue within tomographic data. The subject of the approach is the inter-patient analysis of brain tissue types such as tumor, CSF, white matter, grey matter, bone, fat tissue and background. The class tumor hereby represents the superset of pathological tissue. The analysis pipeline of the presented approach contains feature extraction, classification, twostep texture analysis and morphological postprocessing. Furthermore the paper reports results, that have been reached on the different steps of the pipeline.
\end{abstract}

\section{Introduction}

Segmentation of medical images remains a challenging task, nevertheless imaging techniques have been developed and improved over the last years. As a consequence a variety of approaches were made, in order to segment tomographic image data stemming from computer tomography (CT) or magnetic resonance imaging (MRI). The approaches in general address the identification and distinction of tissue types such as grey and white matter separation as well as the localization of pathological tissue. Reliable and robust results of segmentation algorithms are required by various applications such as irradiation planning, volumetric measurement for tumor regression observation or surface reconstruction for 3D-Visualization. Applying image analysis techniques to multimodal images often involves interactive or semiautomatic techniques [5]. Although there are different approaches that realize an automatic segmentation scheme for tomographic images, they do have common drawbacks: They either rely on a high number of image bands, which results in stressful long acquisition times, or their capability is limited to a small number of detectable anatomical classes, such as cerebospinal fluid $(C S F)$, gray and white matter separation as elaborated in [11].

Another essential aspect for proper evaluation of segmentation algorithms is the extent of required user interaction. Automated segmentation according to Özkan's work[9] requires an interactive setup in a selected slice of the tomographic data set, while the analysis of the subsequent and neighbored slices is performed automatically. In this sense "automated algorithms" were defined to be inter-slice capable. Following this definition the subject of the presented work is to realize a segmentation pipeline that automatically analyzes tomographic data with inter-patient capabilities.

An overview of the segmentation pipeline is given in Figure 1. It was designed to handle tomographic data such as double-echo sequences from magnetic resonance imaging in a pixel based 
approach. It is build of a twostep texture analysis, where step 1 is the mere texture discrimination and step two is the integration of various results into one combined result.

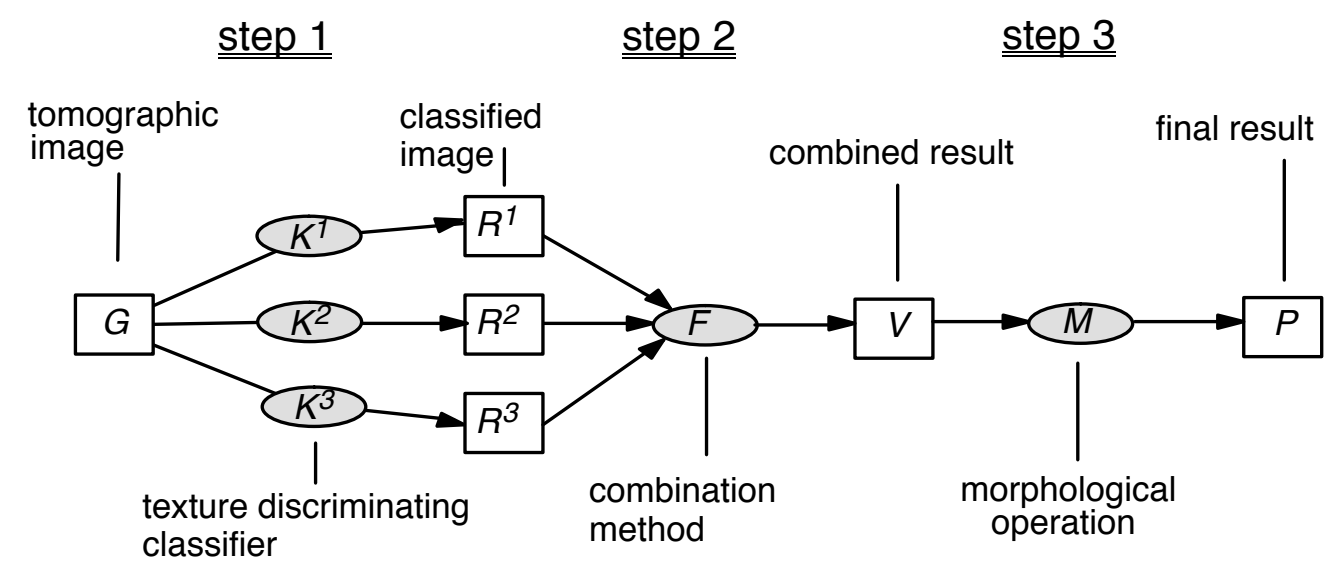

Figure 1. Three-step segmentation pipeline

Even though the data that was used for investigation purposes in this paper did contain additional $c t$-bands, which were matched to the MRI slices [10], the segmentation approach focuses on a small number of multiparametric images. In order to compensate the lack of additional signal intensities a texture discriminating component is employed in the pipeline. Well known classical methods like statistical moments, cooccurrence matrices and textural energy are implemented. A common disadvantage of these methods is, that they work on texture windows of fixed extension. This leads to problems at texture borders, where different texture types adjoin inside a texture window. To reduce this impact a feature extraction method based on the wavelet transform was implemented and investigated recently in a comparative study [3]. The wavelet technique implies multiresolutional properties and therefore allows the description of local and more global characteristics of the texture at the same time. An extensive study has shown, that wavelet features lead to higher classification rates. Unfortunately this also implies an increase in computational expenses.

The embedded classification technique in the segmentation pipeline is the Kohonen Feature Map, that has proven its suitability for image classification in a variety of preceding applications [2]. A self-organized Kohonen Feature Map provides a cluster analysis of the tomographic input data and after additional learning-cycles with Kohonen's LVQ-learning on a training set defined by an expert, it serves as a supervised classifier.

Section 2 of this paper will consider the combination method employed in the pipeline. Results that were made with the additional postclassifying filtering are reported in Section 3 of this paper.

\section{Combination Methods}

To improve the reliability of the classification result the analysis pipeline performs a two-step texture analysis [3], [8]. The basic idea is, that features from different extraction methods are not accumulated in one feature vector. In contrary, for each method an individual classifier is trained (step one of the pipeline) and the results, which were calculated with $n$ different classifiers are combined in a second step. For this purpose a very stringent operation with boolean operators as well as consideration of statistical weights and fuzzy techniques may be used. The additional classification effort with the twostep texture analysis leads to the following advantages: On the one hand the overall classification rates can be improved and on the other hand the final result is much more robust against failures of a single classification method. 


\subsection{Confidential Measure}

A suitable solution for the combination task is the consideration of statistical weights [3] (statistical linking) or the employment of a fuzzy system [7]. Both rely on an assumption for the reliability of each contributing classification result. This reliability can be quantified using a confidential measure $p_{c l s}^{k}$ for the associated classifier $k$.

$$
p_{c l s}^{k}=\frac{R P}{(R P+F N)} \frac{R P}{(R P+F P)}
$$

where $R P$ (right-positive), $F N$ (false-negative) and $F P$ (false-positive) can be derived from the known confusion matrices of the individual classification method $k$. Furthermore the measure $p_{c l s}^{k}$ is calculated class-specificly to class $c l s$, and used as bias for statistical linking or scaling of single tones within a fuzzy system. Figure 2 gives an example for a derived fuzzy set, where membership rates rely on the confidential measure.

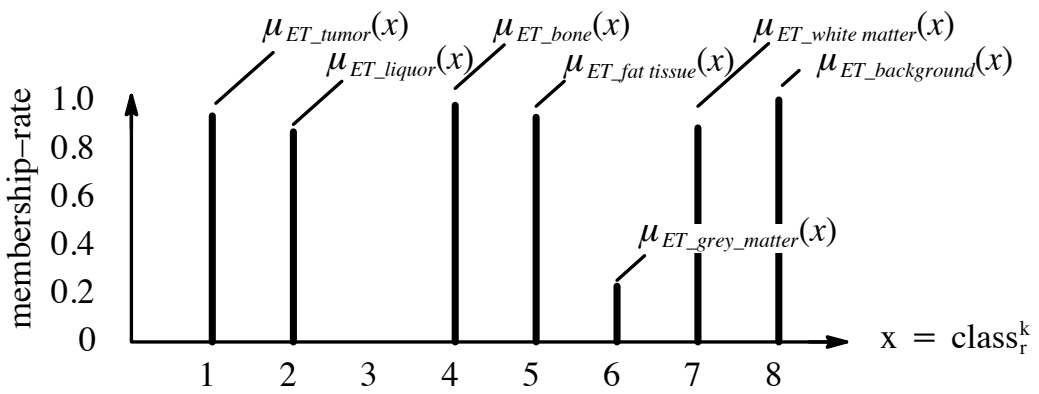

Figure 2. Singleton-Set of a classifier $k$ for fuzzy combination of classification results

More details about fuzzy systems such as the fuzzification component, inference engine, and defuzzification component, as well as the design of appropriate rules can't be explained here in detail, but can be found in the literature [7].

\subsection{Combination Results}

The improvement reached by the combination method has two aspects: First confusion matrices, that describe classification rates of the combined results show fewer confusions than the original input results. Second, one can state, that the amount of misclassified pixels can be reduced significantly.

The outlined segmentation pipeline was tested on over 70.000 test samples stemming from 11 patients with identical pathology. The investigated data sets (with $T_{2}$-weighted band, $p w$-weighted band and $c t$-band) were classified with different classifiers. A comparative study concerning the associated feature extraction methods can be found in [3]. The mentioned classification result now serves as data input for a fuzzy combination component, were the rules were generated on the reference data. In addition a linear separation of the $c t$-band was made, which provides a classification of the bone structure. 
test samples of defined tissue classes

\begin{tabular}{|l|r|r|r|r|r|r|r|r|}
\hline & STAT & tumor & CSF & bone & $\begin{array}{l}\text { fat } \\
\text { tissue }\end{array}$ & $\begin{array}{l}\text { grey } \\
\text { matter }\end{array}$ & $\begin{array}{l}\text { white } \\
\text { matter }\end{array}$ & $\begin{array}{l}\text { back- } \\
\text { ground }\end{array}$ \\
\hline STAT & $\mathbf{0 , 0 0 \%}$ & $9.37 \%$ & $27.01 \%$ & $0,28 \%$ & $0,14 \%$ & $6,73 \%$ & $3,40 \%$ & $0,00 \%$ \\
\hline tumor & $0,00 \%$ & $\mathbf{8 9 , 8 4 \%}$ & $3,61 \%$ & $0,00 \%$ & $0,03 \%$ & $0,00 \%$ & $0,05 \%$ & $0,00 \%$ \\
\hline CSF & $0,00 \%$ & $0,79 \%$ & $\mathbf{6 7 , 7 0 \%}$ & $0,00 \%$ & $0,00 \%$ & $0,00 \%$ & $0,00 \%$ & $0,00 \%$ \\
\hline bone & $0,00 \%$ & $0,00 \%$ & $0,00 \%$ & $\mathbf{9 9 , 4 6 \%}$ & $5,86 \%$ & $0,95 \%$ & $0,00 \%$ & $0,31 \%$ \\
\hline fat tissue & $0,00 \%$ & $0,00 \%$ & $0,06 \%$ & $0,01 \%$ & $\mathbf{9 2 , 7 3 \%}$ & $0,00 \%$ & $0,00 \%$ & $0,00 \%$ \\
\hline grey matter & $0,00 \%$ & $0,00 \%$ & $1,55 \%$ & $0,24 \%$ & $0,61 \%$ & $\mathbf{7 9 , 4 4 \%}$ & $0,78 \%$ & $0,00 \%$ \\
\hline white matter & $0,00 \%$ & $0,00 \%$ & $0,06 \%$ & $0,00 \%$ & $0,64 \%$ & $12,88 \%$ & $\mathbf{9 5 , 7 7 \%}$ & $0,00 \%$ \\
\hline background & $0,00 \%$ & $0,00 \%$ & $0,00 \%$ & $0,00 \%$ & $0,00 \%$ & $0,00 \%$ & $0,00 \%$ & $\mathbf{9 9 , 6 9 \%}$ \\
\hline
\end{tabular}

Figure 3. Confusion matrix for combined classification result

The overall confusion matrix of the combination method is given in Figure 3. The class STAT hereby contains all pixels, that were rejected by the combination method. Rejection occurs if none of the designed fuzzy rules matches the problem due to contradicting class assignments in the input classification results.

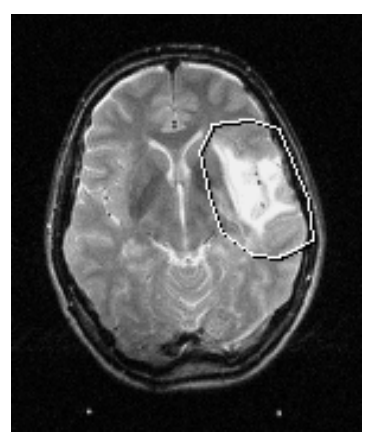

Figure 4. $T_{2-\text { weighted }}$ band from original data set

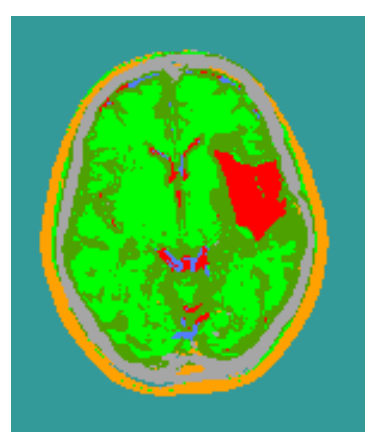

Figure 5. Classification result of Figure 4

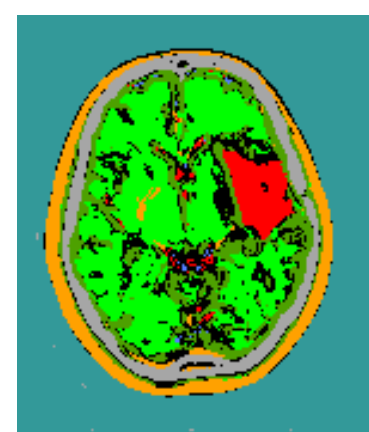

Figure 6. Combined result of Figure 5 and others

The above Figures show the results of step one and step two of the segmentation pipeline. In Figure 4 the $T_{2}$-weighted band from the original data set is shown. A classification result employing a specific feature extraction method (textural energy) is given in Figure 5. Misclassifications, especially confusions between tumor and CSF can often be observed inside the ventricular system. These classification errors are identified and eliminated by the combination method in step 2 of the segmentation pipeline. In the combined result (see Figure 6) only a limited number of misclassified tumor pixels outside the pathological area remain. For reference purpose the isoline definition, that was laid out for irradiation planning, is visualized in Figure 4 marking the pathological area.

\section{Postprocessing}

Combined result-images are postprocessed in this segmentation pipeline applying morphological operations. This postclassifying filtering is necessary, since the combination result from Figure 6 is achieved with an pixel-oriented approach and can not yet be considered to be a segmentation. Within this work the following characteristics, which are essential for further processing, shall define an image segmentation:

An image is devided in a distinct number of homogenous regions, where each region is assigned to a tissue type as anatomical class. Isolated misclassified pixels are re- 
moved such that contour descriptions of the regions will lead to a limited number of contour lines.

In order to fulfill this definition removal of isolated pixels and closing of regions are required. For this postclassifying filtering consecutive operations with binary operators [4] are not suitable, since they neglect anatomical neighborhood. To remedy this disadvantage morphological operations were developed for color-coded images [1] and are now applied in this work.

\subsection{Morphological Operations on Color-Coded Images}

The main intention of the modified operations is, to perform the operation class-overlapping. This concept allows to consider more than one class (color-code) in one processing step and moreover verifies the classification result according to semantic rules, which are deduced from basic anatomic knowledge. As an example Figure 7 uses a sensitive dilation $A \oplus_{s(G)} B$ in order to remove misclassified $C S F$-pixels stemming from mixture effects inside the texture window during texture feature extraction (see [1] for exact definition).

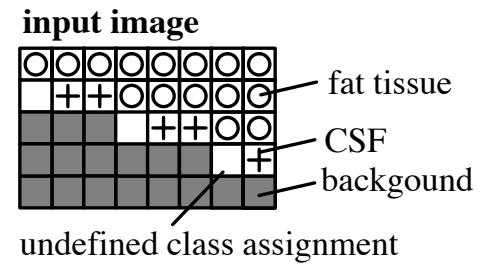

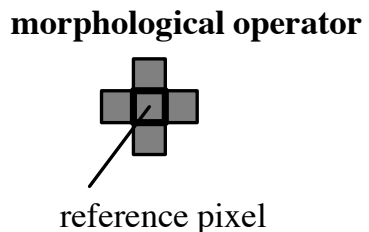

reference pixel

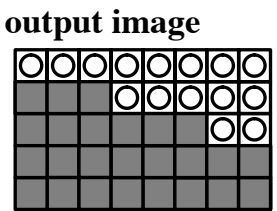

Figure 7. Sensitive dilation on classified images

The operation works sensitive (identified by Index $s$ ) with respect to a defined set $G$ of classes, where in the given example the classes fat tissue and bone are member of $G$. In that case the morphological operator $B$ performs the operation on all background pixels of $A$ and according to the definition will overwrite with its operator elements all color-codes (class assignments of $A$ ) that are not members of the protected set $G$.

\subsection{Segmentation Results}

Morphological postprocessing can significantly improve the classification rates. This holds for the overall classification rates of segmented images, as well as for their visual impression. All datasets, that were analyzed after step two of the segmentation pipeline were processed with step 3 of the pipeline and evaluated afterwards according to the test data set.

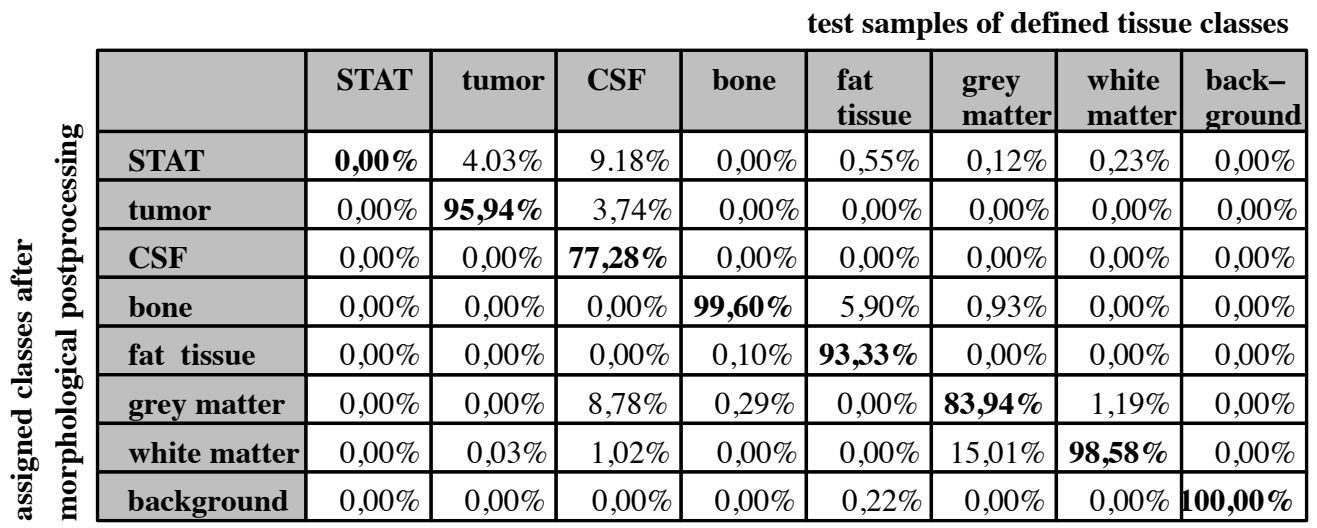

Figure 8. Confusion matrix for segmentation result 
The confusion matrix in Figure 8 illustrates the improvement of classification rates, since most of pixels with an undefined class assignment were labeled with the correct class by the postprocessing operation. The improvement towards an image segmentation in the above sense is also documented with the output images in Figure 9. It can be stated, that all misclassified isolated pixels from Figure 6 were removed and homogenous regions with smoothed region borders were formed by the operation. This final segmentation identifies a pathological area as closed region.

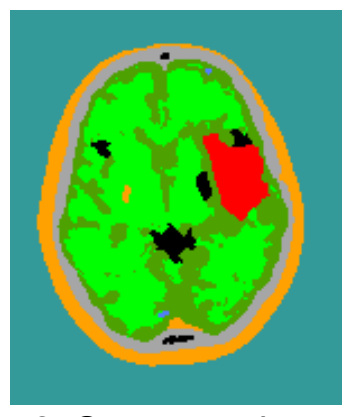

Figure 9. Segmentation result

\section{Conclusion}

This work addresses the automatic segmentation of tomographic images, which is performed in a three-step processing pipeline. While step one of the pipeline implements texture discrimination methods for pixel based classification, step two combines result images and thus improves the reliability of prior decisions. A final postprocessing is performed and eliminates classification errors according to anatomical rules.

The generalization capabilities of the approach was tested on the consideration of more than 70.000 test samples, which were used for statistical evaluation. The results given in this paper show, that segmentation of pathological tissue such as brain tumors can be performed with expected classification rates of approx. 96\%. Thus a robust distinction from 6 other brain tissue classes were made. This evaluation demonstrates, that in contradiction to Özkan [9] inter-patient analysis of tomographic data can be realized.

\section{Acknowledgment}

This research was made possible by the financial support from Deutsche Telekom as part of the project KAMEDIN. Further thanks to he department of Biophysics and Medical Radiation Physics of the German Cancer Research Center, who kindly provided the datasets used in this work.

\section{References}

[1]. C. Busch, M.Eberle,"Morphological Operations for Color-Coded Images", Computer Graphics forum, Vol.14, No.3, Proceed. of EUROGRPAHICS'95, pp.C193-C204, (1995)

[2]. C. Busch, M. Gross: 'Interactive Neural Network Texture Analysis and Visualization for Surface Reconstruction in Medical Imaging", Computer Graphics forum, Vol.12, No.3, Proceed. of EUROGRAPHICS'93, pp.C49-C60, (1993)

[3]. C. Busch : "Wavelet Based Texture Segmentation of Multi-Modal Tomographic Images", to be published in: Computer \& Graphics, vol.21, no.3, (1997)

[4]. G. Gerig, W. Kuoni, R. Kikinis, O. Kübler: "Medical Imaging and Computer Vision: An integrated approach for diagnosis and planning" in: Mustererkennung 1995, GI Informatik aktuell, Springer, pp.425-432, (1989).

[5]. K.H. Höhne et al., "3D Visualization of Tomographic Volume Data using the Generalized Voxel Model". The Visual Computer 6, no. 1, 28-36, (1990)

[6]. T. Kohonen: "The Self-Organizing Map", Proceedings of the IEEE, Vol. 78, No. 9, pp. 1464-1480, (1990)

[7]. B. Kosko: "Neural Networks and Fuzzy Systems", Prentice Hall, (1992)

[8]. A. Krogh, J. Vedelsby: "Neural Network Ensembles, Cross Validation, and Active Learning", in Proceedings NIPS 7, G. Tesauro et al. (eds.), Advances in Neural Information Processing Systems, pp.231-238, MIT Press, (1995)

[9]. M. Özkan, B.M. Dawant, R.J. Maciunas: "Neural-Network-Based Segmentation of Multi-Modal Medical Images: A Comparative and Prospective Study", IEEE Transacions on Medical Imaging, vol.12, no.3, pp.534-544, (1993)

[10]. L.R. Schad, Correction of Spatial Distortion in Magnetic Resonance Agiography for Radiosurgical Treatment Planning of Cerebral Arteriovenous Malformations. Magnetic Resonance Imaging 10, 609-621, (1992)

[11]. C. Tsai, B.S. Manjunath, R. Jagadeesan, ”Automated Segmentation of Brain MR Images". Pattern Recognition, Elsevier Science, vol. 28, no. 12, pp. 1825-1837, (1995) 